\title{
Prevalence of Macular Abnormalities Identified Only on Optical Coherence Tomography in Korean Patients Scheduled for Cataract Surgery
}

\author{
Seo-Yeon Hong, Seonjoo Kim, So-Hyang Chung \\ Department of Ophthalmology, Seoul St. Mary's Hospital, The Catholic University of Korea College of Medicine, Seoul, Korea
}

Purpose: To assess the prevalence of macular abnormalities identified only on macular optical coherence tomography (OCT) which were not suspected by biomicroscopic fundus examination, and examine the clinical outcome of patients with these macular abnormalities during preoperative evaluation for cataract surgery in a large series of Korean patients.

Methods: Macular OCT was performed on patients scheduled for routine cataract surgery by the same physician at Seoul St. Mary's Hospital, between June 2018 and November 2019. The patients' medical records were reviewed retrospectively to obtain demographic data and the results of preoperative evaluation before cataract surgery. Patients were divided into two groups based on the preoperative macular OCT results: normal and abnormal OCT groups.

Results: Nine hundred eighty-seven eyes (698 patients) were included in this study. Macular OCT identified abnormalities in 44 eyes (4.5\%) of 35 patients (5.0\%). Twenty-one eyes (2.1\%) had age-related macular degeneration, 20 eyes (2.0\%) had epiretinal membrane, and three eyes (0.3\%) had lamellar hole. Patients with macular abnormalities identified on macular OCT had a statistically significant higher mean age than those who had normal OCT findings $(p<0.001)$. Best-corrected visual acuity was worse in patients with abnormal macular OCT after cataract surgery $(p=0.048)$.

Conclusions: In the preoperative evaluation for cataract surgery in Korean patients, one in every 20 patients had macular abnormalities identified only on macular OCT in spite of unremarkable macular findings on biomicroscopic funduscopy. Age was significantly higher in patients with abnormal macular OCT findings. Thus, inclusion of macular OCT examination in preoperative screening before routine cataract surgery would be beneficial.

Key Words: Cataract extraction, Diagnostic imaging, Optical coherence tomography

Cataract is a common cause for vision loss worldwide leading to cataract surgery as one of the most commonly performed clinical procedure in the medical field [1]. With

Received: May 27, 2020 Final revision: January 5, 2021

Accepted: February 17, 2021

Corresponding Author: So-Hyang Chung, MD, PhD. Department of Ophthalmology, Seoul St. Mary's Hospital, The Catholic University of Korea College of Medicine, 222 Banpo-daero, Seocho-gu, Seoul 06591, Korea. Tel: 82-2-2258-6203, Fax: 82-2-2258-1196, E-mail: chungsh@catholic.ac.kr significant advancements in phacoemulsification and intraocular lens technology in the last few decades, patients undergoing cataract surgery now have increased expectations regarding visual outcomes [2]. To meet these expectations, preoperative knowledge of underlying ocular condition that may limit the visual gain after surgery, especially in cases with macular pathology, is important [3].

Historically, a dilated biomicroscopic fundus examination was the standard screening method for preoperative evaluation of cataract surgery, but it remains a challenge in 
the presence of media opacities. Now, optical coherence tomography (OCT) is used commonly as a noninvasive and sensitive test for the evaluation of macular structure [4]. Improvements in identifying macular abnormalities and understanding the visual prognosis of these cases confer a substantial benefit to both patients and surgeons.

Prior studies reported that in $7.2 \%$ to $54.2 \%$ of patients, macular pathology was identified on OCT during preoperative evaluation for cataract surgery [5-9]. The purpose of the present study was to assess the prevalence of macular abnormalities identified only on macular OCT, which were not suspected previously by biomicroscopic fundus examination alone in a large series of Korean patients who underwent preoperative evaluation for cataract surgery.

\section{Materials and Methods}

This study included patients who were scheduled for cataract surgery by the same physician (SHC) at Seoul St. Mary's Hospital (Seoul, Korea), between June 2018 and November 2019. Excluded were eyes with a previous diagnosis of macular disease, eyes with precluded funduscopy or macular OCT examination due to media opacity, and eyes with a suspicious biomicroscopic fundus examination. The study followed the tenets of the Declaration of Helsinki. Approval was obtained from the institutional review board of the Seoul St. Mary's Hospital, The Catholic University of Korea (2020-1362-0002). Written informed consent was waived due to the retrospective nature of the study.

The patients' medical records were reviewed retrospectively to obtain demographic data and the results of preoperative evaluation. Preoperative evaluation included slitlamp biomicroscopy to assess the anterior segment, dilated biomicroscopic fundus examination, optical biometry, corneal topography, specular microscopy, and macular OCT. Biomicroscopic fundus examination was done by a retinal specialist. Macular OCT was performed using a Swept-Domain OCT device (Heidelberg Spectralis; Heildelberg Engineering, Heidelberg, Germany) or a Swept-Source OCT device (DRI Triton; Topcon, Tokyo, Japan) by an experienced operator. The scans were also reviewed by a retinal specialist.

Statistical analysis was performed using IBM SPSS Statistics ver. 22.0 (IBM Corp., Armonk, NY, USA). Categori- cal variables are expressed as absolute and relative frequencies. Continuous variables are expressed as the mean \pm standard deviation ( $95 \%$ confidence intervals). The Mann-Whitney $U$-test and the chi-square test were used to compare variables between different groups. For all tests, a $p$-value of less than 0.05 was used to reject the null hypothesis.

\section{Results}

Of the 799 patients (1,126 eyes) scheduled for routine cataract surgery, 48 patients (59 eyes) were excluded owing to previously diagnosed macular abnormality, 29 patients (38 eyes) were excluded owing to precluded funduscopy or macular OCT examination, and 24 patients (42 eyes) were excluded owing to suspicious biomicroscopic funduscopic findings. Thus, 698 patients (987 eyes) were included in

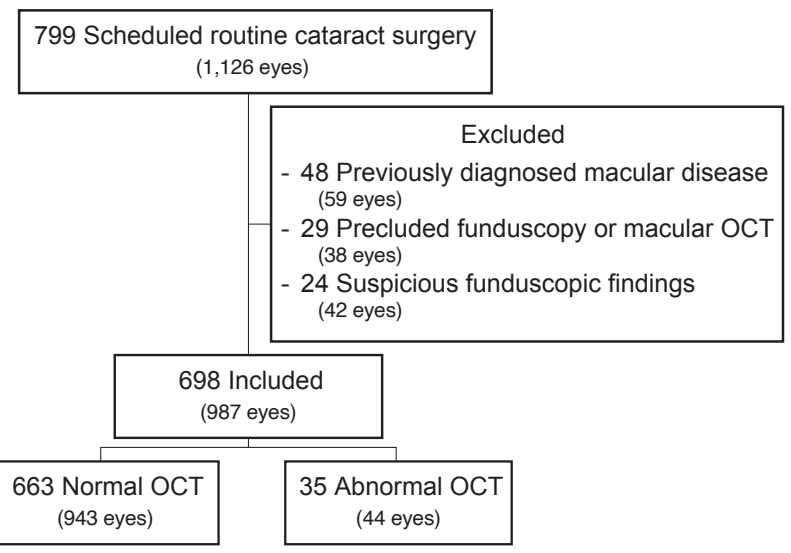

Fig. 1. Screening of patients scheduled to undergo routine cataract surgery. $\mathrm{OCT}=$ optical coherence tomography.

Table 1. Basic demographic data

\begin{tabular}{lc}
\hline Characteristics & Value \\
\hline Female & $410(58.7)$ \\
Age $(\mathrm{yr})$ & $66.3 \pm 11.1$ \\
Diabetes & $131(18.8)$ \\
Hypertension & $198(28.5)$ \\
Heart disease & $24(3.4)$ \\
Cerebrovascular disease & $16(2.3)$ \\
\hline
\end{tabular}

Values are presented as number $(\%)$ or mean \pm standard deviation. 
this study (Fig. 1).

The mean age of the patients was $66.3 \pm 11.1$ years. Of the patients, 410 patients (58.7\%) were women, 131 (18.8\%) had diabetes, 198 (28.5\%) had hypertension, 24 (3.4\%) had heart disease, and 16 (2.3\%) had cerebrovascular disease (Table 1).

Of the 698 patients (987 eyes) included in this study, 663 patients (943 eyes) had normal OCT and 35 patients (44 eyes) had abnormal OCT findings in at least one eye (Fig. 1). With regard to the identified macular abnormalities, 17 patients $(2.4 \%)$ had age-related macular degeneration, 16 patients $(2.3 \%)$ had epiretinal membrane, and two patients $(0.3 \%)$ had lamellar hole (Table 2 and Fig. 2A-2F).

Demographic data of patients with a normal macular OCT and those with an abnormal macular OCT were com-

Table 2. Macular abnormalities identified only on macular optical coherence tomography with unremarkable macular findings on funduscopy

\begin{tabular}{lrr}
\hline Abnormality & Patient & Eye \\
\hline Age-related macular degeneration & $17(2.4)$ & $21(2.1)$ \\
Epiretinal membrane & $16(2.3)$ & $20(2.0)$ \\
Lamellar hole & $2(0.3)$ & $3(0.3)$ \\
Total & $35(5.0)$ & $44(4.5)$ \\
\hline
\end{tabular}

Values are presented as number (\%). pared. Patients with macular abnormalities identified only on macular OCT had a statistically significant higher mean age than those who had a normal macular OCT $(p<0.001)$. There was no statistically significant difference in other characteristics between the two groups. Also, due to the multivariate logistic regression analysis, age was the only statistically significant related risk factor for OCT abnormality ( $p=0.001)$. As the patient gets 1 year older, the odds increase 1.08 times and the $95 \%$ confidence interval for the odds ratio was 1.03 to 1.12 (Table 3). Age stratified

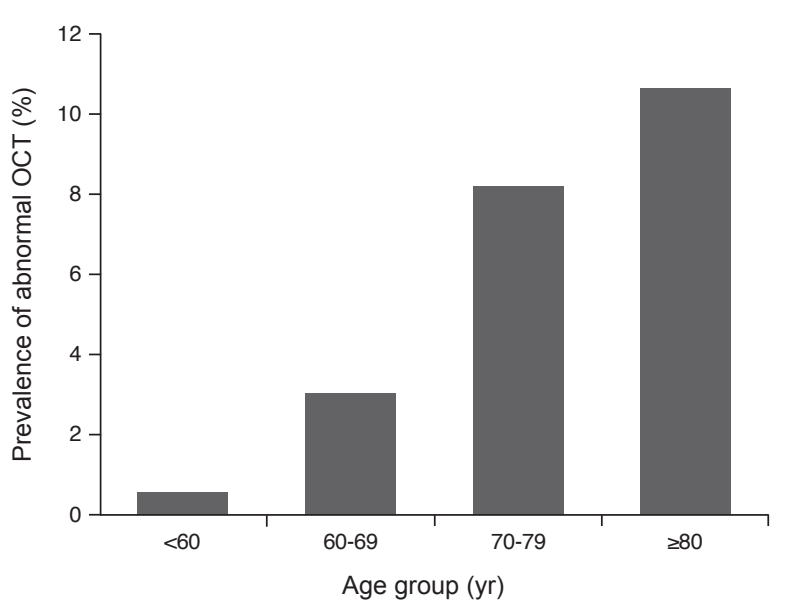

Fig. 3. Age stratified prevalence of abnormal macular optical coherence tomography (OCT) before routine cataract surgery.
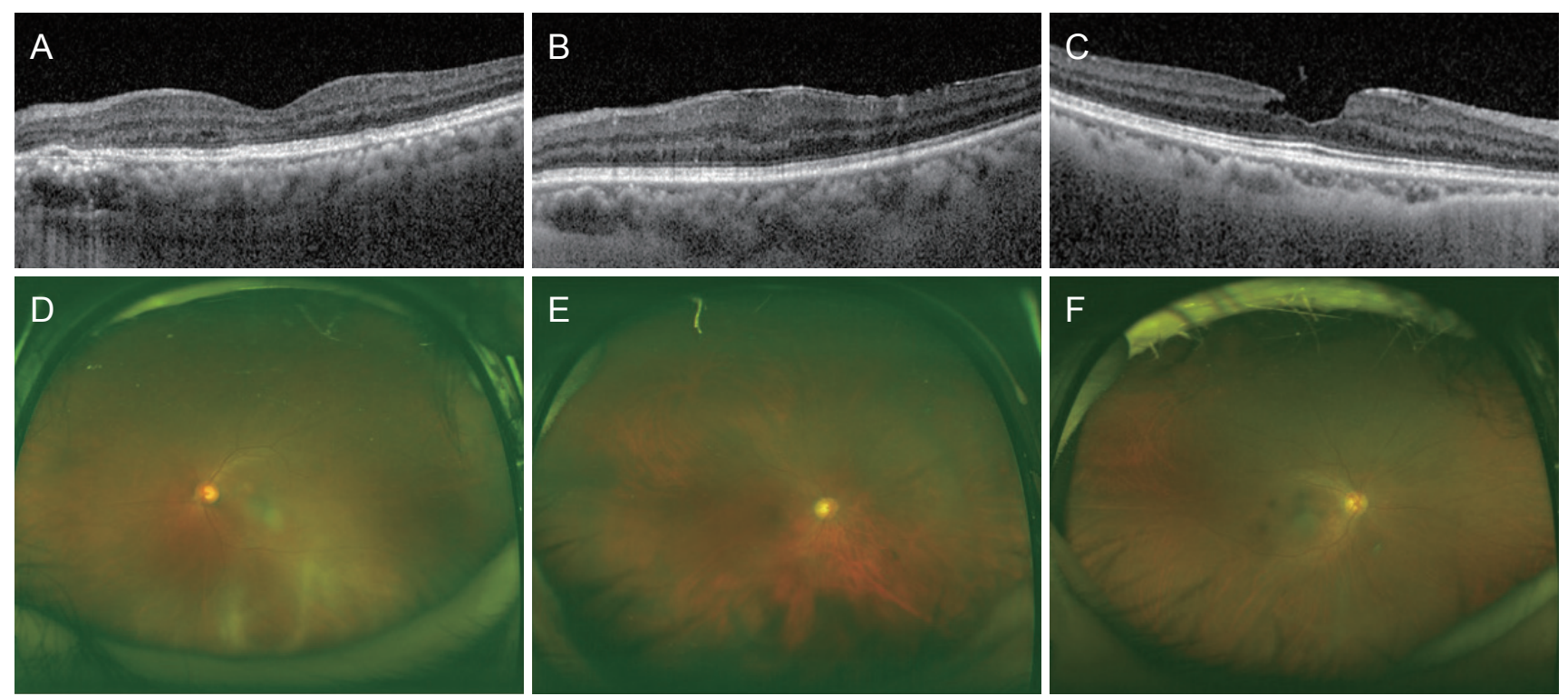

Fig. 2. Macular optical coherence tomography showing (A) age-related macular degeneration, (B) epiretinal membrane, and (C) lamellar hole. Fundus photo showing (D) age-related macular degeneration, (E) epiretinal membrane, and (F) lamellar hole. 
Table 3. Comparison of the demographic data of patients with normal and abnormal macular OCT

\begin{tabular}{|c|c|c|c|c|c|c|}
\hline \multirow{2}{*}{ Characteristics } & \multirow{2}{*}{$\begin{array}{l}\text { Normal OCT } \\
\text { (663 patients) }\end{array}$} & \multirow{2}{*}{$\begin{array}{l}\text { Abnormal OCT } \\
\text { (35 patients) }\end{array}$} & \multirow{2}{*}{$p$-value ${ }^{*}$} & \multicolumn{3}{|c|}{ Multivariate logistic regression } \\
\hline & & & & OR & $95 \% \mathrm{CI}$ & $p$-value \\
\hline Female & $391(59.0)$ & $19(54.3)$ & 0.402 & 0.67 & $0.33-1.37$ & 0.282 \\
\hline Age (yr) & $66.0 \pm 11.1$ & $72.5 \pm 9.4$ & $<0.001^{\dagger}$ & 1.08 & $1.03-1.12$ & $0.001^{\dagger}$ \\
\hline Diabetes & $122(18.4)$ & $9(25.7)$ & 0.397 & 1.14 & $0.49-2.67$ & 0.670 \\
\hline Hypertension & $184(27.8)$ & $14(40.0)$ & 0.154 & 1.20 & $0.56-2.58$ & 0.692 \\
\hline Heart disease & $23(3.5)$ & $1(2.9)$ & 0.888 & 0.51 & $0.06-4.01$ & 0.601 \\
\hline Cerebrovascular disease & $15(2.3)$ & $1(2.9)$ & 0.778 & 0.88 & $0.11-7.08$ & 0.888 \\
\hline
\end{tabular}

Values are presented as number $(\%)$ or mean \pm standard deviation.

$\mathrm{OCT}=$ optical coherence tomography; $\mathrm{OR}=$ odds ratio; $\mathrm{CI}=$ confidence interval.

"Mann-Whitney test, otherwise chi-square test; ${ }^{\dagger} p<0.05$.

Table 4. Preoperative and postoperative visual acuity of patients with normal and abnormal macular OCT

\begin{tabular}{|c|c|c|c|}
\hline & Normal OCT (943 eyes) & Abnormal OCT (44 eyes) & $p$-value \\
\hline \multicolumn{4}{|l|}{ UCVA (logMAR) } \\
\hline Preoperative & $0.44 \pm 0.37$ & $0.52 \pm 0.37$ & $0.022^{\dagger}$ \\
\hline Postoperative & $0.12 \pm 0.20$ & $0.24 \pm 0.24$ & $<0.001^{\dagger}$ \\
\hline$p$-value ${ }^{\ddagger}$ & $<0.001^{\dagger}$ & $<0.001^{\dagger}$ & \\
\hline Preoperative - postoperative & $0.32 \pm 0.32$ & $0.28 \pm 0.31$ & 0.180 \\
\hline \multicolumn{4}{|l|}{ BCVA (logMAR) } \\
\hline Preoperative & $0.41 \pm 0.34$ & $0.37 \pm 0.30$ & 0.745 \\
\hline Postoperative & $0.11 \pm 0.18$ & $0.16 \pm 0.21$ & $0.048^{\dagger}$ \\
\hline$p$-value ${ }^{\star}$ & $<0.001^{\dagger}$ & $<0.001^{\dagger}$ & \\
\hline Preoperative - postoperative & $0.30 \pm 0.30$ & $0.21 \pm 0.19$ & 0.054 \\
\hline
\end{tabular}

Values are presented as mean \pm standard deviation.

$\mathrm{OCT}=$ optical coherence tomography; UCVA = uncorrected visual acuity; logMAR = logarithm of the minimum angle of resolution; $\mathrm{BCVA}=$ best-corrected visual acuity.

"Mann-Whitney test; ${ }^{\dagger} p<0.05$; ${ }^{*}$ Wilcoxon signed rank test.

analysis of the prevalence of abnormal macular OCT showed greater prevalence in groups with elderly patients, as $0.06 \%$ in patients younger than $60,3.13 \%$ in $60 \mathrm{~s}, 8.27 \%$ in $70 \mathrm{~s}$, and $10.71 \%$ in patients older than 80 . A linear-by-linear association test showed a significant increase in prevalence of abnormal macular OCT with increasing age $(p<0.001)$ (Fig. 3).

The visual acuity was also compared between the two groups. Uncorrected visual acuity (UCVA) before cataract surgery was significantly worse in patients with abnormal macular OCT ( $p=0.022)$. Best-corrected visual acuity (BCVA) before cataract surgery was better in patients with abnormal macular OCT, but was not statistically signifi- cant $(p=0.745)$. Postoperative UCVA and BCVA were both significantly worse in patients with abnormal macular OCT ( $p<0.001, p=0.048$, respectively). Changes between preoperative and postoperative UCVA and BCVA were greater in patients with normal OCT compared to patients with abnormal OCT, but was not statistically significant (Table 4).

\section{Discussion}

As the technology developed in the field of cataract surgery, the accessibility and expectancy for cataract surgery 
has increased throughout the decade [2]. Traditionally, a biomicroscopic fundus examination has been accepted as the standard of assessing macular abnormalities in patients scheduled for cataract surgery [3,7]. Now, macular OCT is a noninvasive examination that has great accuracy in diagnosing macular alterations, and some authors have advocated the inclusion of macular OCT in the preoperative evaluation for cataract surgery [5-10]. Furthermore, with an increase in the usage of advanced-technology intraocular lenses, such as multifocal or toric intraocular lenses, and the expectations of patients regarding their visual outcome after cataract surgery, the importance of reliable preoperative evaluation of macular function by OCT has been increased [11]. Thus, we evaluated the prevalence of macular abnormalities that would have not been identified solely by the biomicroscopic funduscopy, and the difference in clinical visual outcomes of patients with abnormal macular OCT in a large series of Korean patients who were scheduled for routine cataract surgery.

Macular abnormalities identified only on macular OCT account for $5.0 \%$ of patients in this study. It means that one in every 20 patients scheduled for routine cataract surgery, might have had an undiagnosed macular abnormality if the preoperative macular screening was performed exclusively by biomicroscopic fundus examination. Similarly, Pinto et al. [5] and Zafar et al. [7] reported that the prevalence of macular abnormalities identified by macular OCT with unremarkable findings in biomicroscopic examination before cataract surgery was $7.2 \%$ and $10.9 \%$, respectively. Furthermore, the most common macular abnormality identified in this study was age-related macular degeneration accounting for $2.4 \%$ of patients $(2.1 \%$ of eyes), followed by epiretinal membrane accounting for $2.3 \%$ of patients ( $2.0 \%$ of eyes) included in this study. Previous studies corroborate our findings, indicating age-related macular degeneration (3.2\%) in of Zafar et al. [7]'s study and epiretinal membrane $3.3 \%$ and $11.05 \%$ respectively) in Pinto et al. [5] and Huang et al. [6]'s studies as the most common macular abnormality. Moreover, among the 35 patients with an abnormal OCT finding, both eyes in 18 patients were included in this study, and 11 patients (61.1\%) also had macular abnormalities in the fellow eye. Thus, patients with macular abnormalities in the other eye have more necessity for macular OCT as preoperative screening before cataract surgery.

We also compared the demographic data of patients ac- cording to their macular OCT examination, and found out that the patients' mean age was statistically higher in those with occult macular diseases. As the patients get older, the prevalence of abnormal macular OCT seems to increase. The other variables we studied did not show a statistically significant correlation with macular diseases. Our results agrees with those of Pinto et al. [5]'s study, in which age was reported to be a statistically significant demographic factor, and differ from Klein et al. [8]'s findings, in which statistically more macular abnormalities were seen in men and patients with heart disease. The disparity between these studies is possibly due to the different populations and sample sizes. The limitation of our study is its retrospective nature and it did not include other modifiable risk factors as smoking, diet, etc.

The clinical significance of preoperative macular OCT was examined by comparing visual outcomes between the normal and abnormal macular OCT groups. Patients with abnormal macular OCT showed worse UCVA and BCVA after surgery. Therefore, we can expect that patients with abnormal macular OCT results in preoperative examination would have a worse visual outcome after surgery, and can inform the patient beforehand about the visual prognosis.

To summarize, in the preoperative examination for routine cataract surgery in a large series of Korean patients, one in every 20 patients had macular abnormalities identified on macular OCT in spite of unremarkable findings on biomicroscopic funduscopy. Age was significantly higher in patients with abnormal macular OCT findings. Moreover, patients with abnormalities in macular OCT showed worse visual outcomes after cataract surgery. Therefore, macular OCT is considered to be a sensitive tool in screening preoperative patients scheduled for routine cataract surgery than undergoing biomicroscopic funduscopy alone, especially in patients over the age of 70 . Preoperative macular OCT can be also beneficial in terms of efficacy and safety by predicting and informing the patients beforehand about their visual outcomes. In conclusion, preoperative screening for macular diseases by not only biomicroscopic funduscopy but also by macular OCT allows patients undergoing and surgeons preforming cataract surgery to understand the visual prognosis and to receive and provide individualized counseling. 


\section{Conflict of Interest}

No potential conflict of interest relevant to this article was reported.

\section{Acknowledgements}

This research was supported by Basic Science Research Program through the National Research Foundation of Korea (NRF) funded by the Ministry of Education (2020R1A2B5B 01002407).

\section{References}

1. Bourne RR, Stevens GA, White RA, et al. Causes of vision loss worldwide, 1990-2010: a systematic analysis. Lancet Glob Health 2013;1:e339-49.

2. Davis G. The evolution of cataract surgery. Mo Med 2016;113:58-62.

3. See CW, Iftikhar M, Woreta FA. Preoperative evaluation for cataract surgery. Curr Opin Ophthalmol 2019;30:3-8.

4. Jacob J, Stalmans P. Prevalence of vitreoretinal interface abnormalities as detected by spectral-domain optical coherence tomography. Ophthalmologica 2016;236:81-7.
5. Pinto WP, Rabello LP, Ventura MC, et al. Prevalence of macular abnormalities identified only by optical coherence tomography in Brazilian patients with cataract. $J$ Cataract Refract Surg 2019;45:915-8.

6. Huang X, Zhang Z, Wang J, et al. Macular assessment of preoperative optical coherence tomography in ageing Chinese undergoing routine cataract surgery. Sci Rep 2018;8:5103.

7. Zafar S, Siddiqui MA, Shahzad R, Shahzad MH. Sweptsource optical coherence tomography to screen for macular pathology in eyes having routine cataract surgery. $J$ Cataract Refract Surg 2017;43:324-7.

8. Klein BR, Brown EN, Casden RS. Preoperative macular spectral-domain optical coherence tomography in patients considering advanced-technology intraocular lenses for cataract surgery. J Cataract Refract Surg 2016;42:537-41.

9. Hirnschall N, Leisser C, Radda S, et al. Macular disease detection with a swept-source optical coherence tomography-based biometry device in patients scheduled for cataract surgery. J Cataract Refract Surg 2016;42:530-6.

10. Moreira Neto CA, Moreira Junior CA, Moreira AT. Optical coherence tomography in patients undergoing cataract surgery. Arq Bras Oftalmol 2015;78:241-5.

11. Braga-Mele R, Chang D, Dewey S, et al. Multifocal intraocular lenses: relative indications and contraindications for implantation. J Cataract Refract Surg 2014;40:313-22. 